\title{
Lo pequeño es hermoso: una visión panorámica de la escuela rural catalana
}

\author{
Lluís Samper, Teresa Sala y Xavier Burrial \\ Universitat de Lleida \\ lluis.samper@geosoc.udl.cat, sala@pip.udl.cat, x.burrial@filcef.udl.cat
}

RESUM

Al pot petit hi ha la bona confitura: una visió panoràmica de l'escola rural catalana

En aquest article pretenem donar una visió general de l'escola rural a Catalunya i del medi sòciogeogràfic en la qual està immersa. En les darreres dècades hi ha hagut canvis en el món rural, que ha deixat de ser essencialment agrícola per transformar-se en un espai residencial, de serveis o bé d'oci. Si tenim en compte els canvis estructurals, però també simbòlics que s'han produït en la societat rural catalana, la percepció que tenim actualment de la ruralitat ja no està associada, com era abans, a la idea d'endarreriment, ignorància, pobresa econòmica o comunicacions deficients. De la mateixa manera, l'escola rural ja no és "la Ventafocs" de l'Administració educativa i, malgrat que representa menys del dos per cent del total dels alumnes de l'ensenyament infantil i primari, gaudeix de professorat qualificat i d'equipaments comparables als de les escoles de ciutat.

Paraules clau

Escola rural, organització escolar, ruralitat, territori, canvi social.

\section{RESUMEN}

\section{Lo pequeño es hermoso: una visión panorámica de la escuela rural catalana}

En este artículo pretendemos ofrecer una visión general de la escuela rural en Cataluña y del medio socio-geográfico en la que está inmersa. En las últimas décadas ha habido cambios en el mundo rural, que ha dejado de ser esencialmente agrícola para transformarse en un espacio residencial, de servicios o de ocio. Habida cuenta de los cambios estructurales, pero también simbólicos que se han producido en la sociedad rural catalana, la percepción que hoy tenemos de la ruralidad ya no está asociada, como en el pasado, a la idea de retraso, ignorancia, pobreza económica o malas comunicaciones, De la misma manera, la escuela rural ya no es "la Cenicienta" de la Administración educativa y, a pesar de representar menos del dos por ciento de los alumnos de la enseñanza infantil y primaria, goza de profesorado cualificado y de equipamientos comparables a los de las escuelas de ciudad.

\section{Palabras clave}

Escuela rural, organización escolar, ruralidad, territorio, cambio social.

\section{Resumé}

\section{Ce qui est petit est joli : une vision panoramique de l'école rurale catalane}

Dans cet article nous prétendons présenter une vision générale de l'école rurale en Catalogne et du milieu socio-géographique dans lequel elle s'insère. Dans les dernières décennies, il y a eu des transformations dans le monde rural, qui n'est plus essentiellement agricole, pour devenir un espace 
résidentiel, de services ou de loisirs. Si nous considérions les changements structurels, mais en même temps symboliques produits dans la société rurale catalane, la perception que nous avons aujourd'hui de la ruralité n'est pas associée comme avant à l'idée de retard, ignorance, pauvreté économique ou de mauvaises communications. De la même façon, l'école rurale n'est plus « la Cendrillon » de l'Administration éducative et bien qu'elle représente moins de deux pour cent des élevés des enseignements préscolaire et primaire, elle bénéfice d'enseignants qualifiés et d'équipements comparables à ceux des écoles urbaines.

\section{MoTS-CLÉ}

École rurale, organisation scolaire, ruralité, territoire, changement social.

\section{Abstract}

\section{Small is beautiful: a panoramic look at the Catalan rural school}

In this paper we pretend to show a general view of the Catalan rural school and of the social and geographical element in which it is immersed. In the last few decades there have been changes in the rural area which is no more substantially agricultural, but it has developed into a residential, services and leisure area. Keeping in mind these structural changes, but symbolic at the same time, which have taken place in the Catalan rural society, nowadays the perception that we have of the rurality is not any more associated, as it was in the past, to the impression of backwardness, ignorance, economic poverty or poor means of transport. In the same way, the rural school is no longer "the Cinderella" of the Educative Administration, and despite representing less than the two per cent of the students of early years and primary school, they have well qualified teachers and facilities similar to the ones of the schools located in the cities.

\section{KeYwords}

Rural school, school organization, rurality, territory, social change.

\section{Introducción}

El grupo de la escuela rural catalana lo constituyen pequeños centros escolares, la mayoría con sólo dos o tres docenas de alumnos. Tampoco cuantitativamente representan una proporción significativa del sistema educativo: menos del dos por ciento del total de los alumnos de la enseńanza infantil y primaria, y aproximadamente una cuarta parte del total de las escuelas públicas. En conclusión, la escuela rural de Cataluña es pequeña... pero no importa pues, como sabemos, lo pequeño es hermoso.

Pero además, en periodos históricos tan concretos como la Segunda República, las postrimerías del franquismo y la transición democrática dichas escuelas han cobijado a importantes movimientos de renovación pedagógica que desde las comarcas rurales aspiraban a catalizar el cambio de modelo educativo.

Por último, la significación simbólica acaecida en las sociedades de la Europa occidental durante las tres o cuatro últimas décadas ha contribuido, por ósmosis, a revalorizar todo lo relativo al mundo rural. En resumen, que la escuela rural catalana aparece rodeada de un aura de ejemplaridad que, tal vez, no siempre corresponda a la realidad.

En este trabajo pretendemos ofrecer una visión panorámica pero, en la medida de nuestras capacidades, documentada y objetiva, de dicho entorno educativo. Nuestro punto de partida, que en 
realidad es nuestra principal hipótesis, es que la renovación en Cataluña ha ido paralela a los cambios sociales (demográficos, económicos, culturales y políticos) que han dado lugar a las nuevas ruralidades. Por ello hemos iniciado nuestra exposición con una breve descripción de nuestro sistema educativo para abordar, con un poco más de detalle, los cambios estructurales, pero también simbólicos que se han producido en la sociedad rural catalana a lo largo de las últimas décadas. Finalizaremos el texto con un resumen de la situación actual de la escolarización rural, con sus luces pero también con sus sombras.

\section{La escuela y su contexto}

Cataluña es una región situada al noreste de España, tiene una extensión de $31.890 \mathrm{~km}^{2}$. Geográficamente es una región muy diversa, con una zona montañosa en el norte y con $750 \mathrm{~km}$. de costa al este, su población representa el $16 \%$ de la población española — siendo la segunda región más poblada - con aproximadamente 7.500.000 habitantes — con una distribución desigual. La población se concentra mayoritariamente en la costa, especialmente en el área metropolitana de Barcelona que agrupa 5,5 millones de habitantes; en contraste, las comarcas del interior y del Pirineo están poco pobladas, con una densidad inferior a 50 habitantes $/ \mathrm{km}^{2}$.

El municipio es la base de la organización territorial de Cataluña. Según datos del Instituto Nacional de Estadística, hay 947 municipios de distintas dimensiones: 482 tenían menos de 1000 habitantes, 119 superan la población como para ser considerados ciudad ${ }^{1}$, de los cuales hay 63 que superan los 20.000 habitantes y en los que reside el $70 \%$ de la población catalana.

El mapa siguiente nos muestra claramente la distribución de la población en Catalunya:

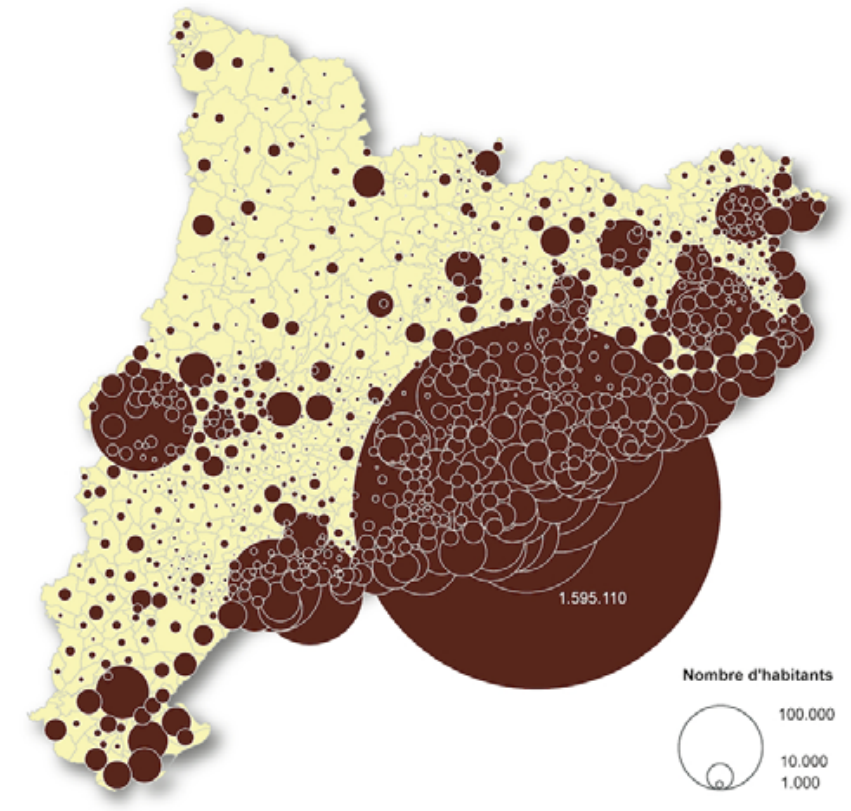

Mapa 1: Distribución de la población en Cataluña. Fuente: Atlas de la nova ruralitat. ${ }^{2}$

1. El criterio que se utiliza habitualmente para definir las ciudades es el estadístico, en España el volumen de población mínimo para considerar a un núcleo de población como ciudad es de 10.000 habitantes.

2. ALDOMÀ i BUIXADÉ, I, Atlas de la nova ruralitat, Lleida, Fundació del Món Rural, 2009, p 13.

Edición en línea: http://www.fmr.cat/atles/materials/atlesruralitat.pdf 
Desde un punto de vista administrativo, España está organizada en Regiones Autónomas que tienen sus propios parlamentos y gobiernos regionales. A distintos niveles, las regiones tienen transferidas diversas competencias. En virtud de su Estatut d'Autonomia de 1979 (en 2006 se aprobó un nuevo Estatuto), la Generalitat de Catalunya (nombre que recibe el gobierno autónomo) tiene transferidas, por ejemplo, competencias en enseñanza universitaria y no universitaria, cultura, sanidad, servicios sociales, policía, etc.

\section{La enseñanza en catalunya}

En el conjunto de España, la enseñanza es obligatoria y gratuita de los seis a los dieciséis años, tanto en los centros públicos como en los centros privados que tienen un concierto con las distintas administraciones educativas. La educación primaria tiene seis cursos y la educación secundaria obligatoria cuatro. Los alumnos pueden seguir estudios post-obligatorios durante dos cursos, bien sea de Formación Profesional o de Bachillerato, dando acceso estos últimos a la universidad. Por otra parte, los centros de Educación Infantil y Primaria acogen alumnos a partir de los tres ańos. En los centros públicos, las etapas no obligatorias también son gratuitas.

Las transferencias en educación no universitaria se produjeron a finales de 1980 (RD 2808/1980 de 3 de octubre, BOE 314 de 31 de diciembre de 1980), con lo cual el gobierno regional se hizo cargo de los centros de enseńanza, de los docentes, de la inspección educativa, pero lo que es más importante, también "de la elaboración de planes de estudio y orientaciones pedagógicas que desarrollen y complementenlas enseńanzas mínimas que establezca el Estado" (apartado B 7. del RD). El Estatuto de 2006 recoge en el artículo 131 la actualización de las transferencias en educación, lo que significa, dicho de otro modo, la capacidad de desarrollar las leyes de educación.

Un hecho diferenciador catalán es la existencia de dos lenguas oficiales: el catalán y el castellano. El Estatuto de Autonomía de Cataluña de 2006, especifica que el catalán es la lengua propia de Cataluña, "es la lengua de uso normal y preferente de las Administraciones públicas y de los medios de comunicación públicos de Cataluña, y es también la lengua normalmente utilizada como vehicular y de aprendizaje en la enseñanza [...] todas las personas tienen derecho a utilizar ambas y los ciudadanos de Cataluña el derecho y el deber de conocerlas" (artículo 6 del Estatut). Cuando terminan la escolarización obligatoria — por noma general a los 16 ańos_-, los alumnos deben tener un perfecto conocimiento de las dos lenguas oficiales. Además, "los poderes públicos deben promover el conocimiento suficiente de una tercera lengua al finalizar la enseñanza obligatoria" (artículo 43.2 del Estatut).

Cuando hablamos de enseñanza en Cataluña, debemos pues tener en cuenta aspectos tan diversos como las dimensiones de los municipios, la localización de los centros de enseñanza o el bilingüismo.

\subsection{La escuela rural en Catalunya, una breve visión histórica}

\section{"Pasa más hambre que un maestro de escuela"}

Los orígenes de la escuela rural debemos buscarlos en la Ley de Instrucción Pública (también conocida como Ley Moyano, del nombre del ministro que la propuso en 1857). En aquella época, España era uno de los países de Europa con una de las tasas más elevadas de analfabetismo. Esta ley proclamó la Enseñanza Primaria obligatoria de los 6 a los 12 ańos, siendo gratuita para aquellos que 
no pudieran pagarla y todos los municipios que tuvieran suficiente matrícula podían disponer de una escuela para niños y otra para nińas. Con pequeńas modificaciones la Ley Moyano perduró en sus aspectos fundamentales hasta la Ley General de Educación de 1970.

La evolución de la enseñanza primaria en el sistema educativo español desde un modelo escolar en el que predominaban las escuelas unitarias "escuelas-aula" de un solo maestro o maestra a otro focalizado en los centros escolares graduados ("escuelas-colegio") se realiza prácticamente a lo largo de todo el siglo XX. Detrás de la morosidad de este cambio pedagógico subyacen obviamente las peculiaridades de unos procesos de modernización prolongados y discontinuos (en concreto, la desigual urbanización del territorio español durante la primera mitad del pasado siglo ${ }^{3}$, pero también debemos considerar las variables administrativas e, incluso, ideológicas. Así mientras para los políticos liberales de la Segunda República, como acérrimos defensores del reformismo pedagógico, la graduación escolar era uno de sus principales objetivos educativos, para el franquismo, en especial durante el periodo anterior al Plan de Estabilización de 1959, la escuela rural encajaba en su ideal de España autártica ${ }^{4}$. Tal vez por ello, se ha tendido a asociar, al menos hasta época reciente, escuela rural con escuela incompleta, dicho de otro modo, con un tipo de escuela no sólo no graduada sino materialmente deficiente y pedagógicamente arcaica, poco organizada y poco eficiente. Los maestros de escuela rural eran por lo general los menos preparados y los peor retribuidos.

\section{"Si cierran la escuela el pueblo se muere"}

La Ley General de Educación de 1970 aceleró el proceso de concentraciones escolares (escuelas graduadas comarcales y escuelas hogar o internados) iniciado en la década anterior. Desde una planificación tecnocrática, el objetivo explícito de este nuevo reformismo educativo en las postrimerías del franquismo no era otro que el de adecuar el modelo escolar a los cambios socioeconómicos (industrialización, desarrollo económico, migraciones interiores y exteriores...) que se suceden ininterrumpidamente en España a partir de los años setenta. En este sentido, el éxodo rural actuó como catalizador de los diversos decretos y resoluciones ministeriales que favorecían la política de concentración escolar pero, al tiempo, la supresión de las pequeñas escuelas mixtas y unitarias favoreció la crisis agraria y el abandono de las pequeñas poblaciones. Para decirlo en palabras de informantes de un estudio etnográficos de la época: "Si cierran la escuela el pueblo se muere" . La política de concentración escolar fue particularmente acusada en las comarcas de población dispersa, especialmente en las del área pirenaica.

3. Uno de los rasgos demográficos más significativos de la evolución reciente de la estructura social española es el enorme trasvase de población entre las áreas rurales y las ciudades. Durante la segunda mitad del siglo XX (significativamente la década que trascurre entre 1955 y 1965) España deja definitivamente atrás su pasado agrario y rural para convertirse en urbana y orientar la actividad hacia los servicios (CAMARERO, L., coord., La población rural de España. De los desequilibrios a la sostenibilidad social, Obra Social de La Caixa, Barcelona, 2009, p.30.)

Según datos del INE, el éxodo rural continúa. Los municipios de menos de 2000 habitantes disminuyeron un 6,2\% su población (de 2.997.457 a 2.811.268) entre 2001 y 2011.

Tampoco podemos ignorar la dimensión simbólica de tales procesos estructurales, es decir, las desiguales y cambiantes valoraciones de los urbanitas hacia los aldeanos y viceversa.

Véase: LISÓN, C. "Aspectos del pathos y ethos de la comunidad rural”. Revista Española de la Opinión Pública, 1976, pp.43, 57-72 y LITTLE, J. y AUSTIN, P. “Woman and rural idyl”, Journal of rural studies, 12, 1996, pp. 101-111.

4. VIÑAO, A., Escuela para todos. Educación y modernidad en la España del s. XX, Marcial Pons Historia, Madrid, 2004, p. 25.

5. GONZÁLEZ BUENO, M. "Situación de la enseñanza en la comarca del Pallars Sobirà, Lérida”. En KNIPMAYER, 


\subsection{El renacer de la escuela rural}

Pero en el caso específico de Cataluña, la fase final de implantación de la L.G.E. coincidió con los primeros traspasos de competencias educativas a las nuevas autoridades autonómicas así como, en el mismo curso escolar de 1979-80, las primeras elecciones municipales democráticas y desde los movimientos de renovación pedagógica de los maestros rurales o en las Jornadas de la Escuela Rural de Cataluña se reivindicó una dignificación de la escuela rural frente a las concentraciones.

En síntesis, se trataba de repensar la escuela rural tal y como estaba organizada y como resultado de este proceso de discusión, elaboración teórica y experimentación práctica, desde la base del movimiento de maestros surgieron iniciativas y propuestas, como la creación de las zonas escolares rurales (ZER) que, en mayor o menor medida, fueron asumidas por las autoridades educativas de la Generalitat de Cataluña. Como contestación al modelo de concentraciones escolares se llegó a demostrar que volver a abrir escuelas en los pueblos no suponía un incremento en la factura de la educación, ya que era más económico desplazar a los maestros a las escuelas que transportar a los alumnos a las escuelas graduadas de referencia. En Cataluña, por ejemplo, el número de alumnos transportados en el curso 1977-78 fue de 35.184, el 9\% del total de los alumnos. Los padres y madres de alumnos y las autoridades locales mostraron su apoyo a las propuestas de los maestros.

Todo ello incidió en un cambio de tendencia en las políticas educativas tanto en lo relativo a frenar las concentraciones escolares como abriendo un debate sobre el presente y futuro de la escolarización rural $^{7}$ Como fruto de todos estos procesos de transformaciones a nivel político y administrativo, pero también de carácter socioeconómico y cultural cambió el concepto de escolarización rural así como su valoración. Lo cierto es que si la escuela rural catalana del último tercio del siglo XX era, para decirlo en palabras de docentes de la época, "una cenicienta de la Administración estatal" y parecía encaminarse a su desaparición, en la actualidad no sólo no ha muerto sino que, gracias en gran parte a sus docentes, goza al parecer de buena salud.

\section{La ruralidad en el siglo $X X I$}

\section{"Ser moderna y de pueblo, a la vez"}

Actualmente, hablar de espacio rural no significa necesariamente hablar de espacio agrícola. Podemos considerar el momento presente y las perspectivas de futuro desde dos puntos de vista en cierto modo contrapuestos y que pueden explicarse por la diversidad de las situaciones existentes. El primero considera la situación de las zonas rurales como áreas que están en crisis, el término que se utiliza es "desertificación" y la perspectiva es el desequilibrio demográfico, el paro, la marginalización de la población que se queda al campo.

Una percepción totalmente opuesta es la que habla de "renacimiento rural" y de ruralidad escogida. Parte de la constatación que hay un crecimiento demográfico en algunas zonas rurales,

GONZÁlEZ BUENO, M. y SAN ROMÁN, T., Escuelas, pueblos y barrios. Antropología educativa, p. 47), Akal, Madrid, 1980.

6. SUBIRATS, M. L'escola rural a Catalunya, Rosa Sensat/Ed. 62, Barcelona, 1983, p 33.

7. SOLER, J., "El maestro y la fisonomía propia de la escuela rural” in LLEVOT, N. Y GARRETA, J. (eds.), Escuela rural y sociedad, Edicions de la Universitat de Lleida, Lleida, 2008, p. 33.

8. VV.AA., L'escola rural a Catalunya. Primeres Jornades Escola Rural, ICE de la UAB, Barcelona, 1980, p. 5. 
sobre todo en las que están cerca de centros urbanos importantes. Kaiser ${ }^{9}$ considera que se está modelando una nueva composición social y que existen posibilidades de renovación, de innovación y de desarrollo.

En nuestras sociedades contemporáneas, aquello que es rural parece estar de moda y, no obstante, la población continúa estando concentrada en las ciudades. Ser de pueblo tiende a ser percibido como una cosa positiva pero nos podríamos preguntar cuáles son las razones de esta supuesta o real revalorización de la ruralidad. ¿¿Será la contrapartida del desarraigo urbano o sólo es otro espejismo identitario? ¿Cómo se define hoy la identidad rural? ¿En qué medida esta moda no forma parte de un proceso más amplio de regreso a lo comunitario? ${ }^{10}$

En contra del tópico, la globalización no es sinónimo de uniformización cultural puesto que paralelamente a los procesos de mundialización, flujos financieros, tecnológicos, mediáticos... surgen procesos de localización, de reconstrucción identitaria, de sentimientos de arraigo, de exacerbación de aquello que es étnico o religioso ${ }^{11}$. Aparece la utilización, cada vez más frecuente del neologismo "glocalitzación”. Esta glocalitzación, así como sus fenómenos concomitantes de desagrarización y movilidad están redefiniendo el concepto del continuo rural-urbano ${ }^{12}$.

El mundo rural se definía por defecto, o mejor por carencia. Si la ciudad era el espacio de vanguardia y progreso, el campo se percibía como el lugar de atraso, el espacio mantenedor de costumbres inútiles e indignas que privaban de libertad de los suyos ${ }^{13}$. Rural, entendido pues como sinónimo de carencia, atraso, tosquedad, aislamiento...

También las ciencias sociales (sociólogos como Tönnies o Weber o antropólogos como Redfield) tendían a subrayar el contraste entre sociedad rural y urbana a partir de una serie de variables — dimensiones y densidad poblacional, actividad económica, ocupación, valores...- que adoptaban un carácter bipolar. De hecho, a mediados del siglo pasado las teorías funcionalistas sobre la modernización defendían una convergencia gradual y progresiva entre las diferentes sociedades humanas hacia un modelo urbano-industrial. Modernidad y desarrollo industrial (industrialización, crecimiento económico, salarización...), político (secularización, democratización, burocratización...) y cultural (alfabetización, desarrollo científico...) eran las dos caras de una misma moneda. En consecuencia, el mundo rural era aquello pre-moderno, es decir aquello que era atrasado económicamente, autoritario o despótico... Justo es decir, en honor a la verdad, que esta visión estereotipada del campo incluía, no obstante, algunos elementos positivos: la idealización bucólica (belleza y sencillez), la armonía comunitaria (orden y sosiego) la exaltación de las raíces y de la autenticidad.

Pero a mediados de la década de los años sesenta esta visión dicotómica e incluso los mismos conceptos de sociedad rural y sociedad urbana entran en cuestión al ser incapaces de explicar los cambios sociales que se están produciendo a las sociedades desarrolladas ${ }^{14}$. En efecto, el espacio

9. KAISER, B., La renaissance Rurale : Sociologie des campagnes du monde occidental, Armand Colin, Paris, 1990.

10. BAUMAN, Z., Comunidad. En busca de seguridad en un mundo hostil, Siglo XXI, Madrid, 2003.

11. BAYART, J.F., L' Illusionidentitaire, Fayard, Paris, 1996.

CASTELLS, M., La era de la información, II. El poder de la identidad. Alianza Editorial, Madrid, 1998.

BAUMAN, Z., Globalització. Les consequències humanes, Pòrtic-UOC, Barcelona 2001.

12. SAMPEDRO, R., "Cómo ser moderna y de pueblo a la vez: los dicursos del arraigo y del desarraigo en las jóvenes rurales" Revista de Estudios de Juventud, nº3 (2008), pp. 179-183.

13. Col-lectiu CCCB 'Forum Seminari Món rural, Món Urbà', Primer Congrés del món Rural a Catalunya, Barcelona: Fundació Món Rural, Dep. d'Agricultura, Ramaderia i Pesca, Generalitat de Catalunya, Barcelona, 2006, p. 449.

14. NEWBBY, H.; SEVILLA-GUZMÁN, E., Introducción a la sociología rural, Alianza Editorial, Madrid,1983, p. 44. 
rural ya no cumple únicamente funciones agrarias sino que empieza a diversificarse en actividades industriales y de servicios y también como apoyo a las nuevas demandas de ocio procedentes de la población urbana. A medida que avanza el proceso de urbanización y se reduce la población que vive en el campo, el mundo rural se presenta como apoyo de una amplia variedad de usos entre los cuales la agricultura ya no es la única ni la principal actividad económica. Paralelamente, en los países desarrollados, ambos espacios, rural y urbano, se complementan y entonces sus diferencias son cada vez menos importantes. La creación de infraestructuras, la disminución real de las distancias, las nuevas tecnologías de la información y la comunicación se traducen en una nueva ordenación del territorio, pasando del contraste a la graduación espacial ${ }^{15}$. Aproximadamente, en la década de los noventa y coincidiendo con la rurubanización, interdependencia entre ambos procesos de urbanización y neo-ruralismo, se empieza a hablar en Europa de la Renaissance rurale ${ }^{16}$ o de Rural $I d y l l^{17}$. Ante la visión negativa del campo, vemos como en estas sociedades de la modernidad tardía, el campo o para decirlo en términos más prosaicos "el ser de pueblo" es sinónimo de calidad de vida, de naturaleza, de identidad, de autenticidad... Lo rural, visto como un reflejo del desasosiego urbano, ofrece un cúmulo de experiencias positivas: desde vivir tranquilo escuchando el ritmo cíclico de la naturaleza hasta la recuperación de unas relaciones humanas verdaderas. Vivir en un pueblo deja de ser un castigo para convertirse en un privilegio.

Por más evidente que sean estos amables estereotipos sobre la ruralidad, es decir, esta visión mitificada del mundo rural, como una especie de arcadia incontaminada se corresponde poco con la imagen que los medios nos ofrecen del campo catalán a principios del siglo XXI: incendios forestales, contaminación de acuíferos, problemas sociales asociados a los jornaleros inmigrantes... De todas maneras, quizás si focalizamos nuestra atención en el análisis de los contenidos simbólicos de la publicidad, los materiales escolares, e incluso algunos discursos políticos podemos encontrar indicios de una cierta recuperación de ideologías de "regreso a las raíces" ${ }^{18}$ Pero, antes de adentrarnos en el tempestuoso territorio de los imaginarios colectivos, veamos algunos datos sobre los cambios sociales en el mundo rural catalán en los últimos veinte o treinta últimos ańos.

Podemos analizar los niveles de ruralidad a partir de criterios objetivos como por ejemplo el porcentaje de población activa agraria, el tamaño y la densidad de la población de los municipios, el PIB del sector primario, o... y los resultados, a pesar de que con matizaciones, confirman el descenso demográfico y económico del territorio rural. Por ejemplo, si en 1981 la población activa agraria representaba el 8,7 \% de la fuerza de trabajo del conjunto de Cataluña, veinte años después era únicamente del 2,46\%. Ya en 2001, el PIB del sector primario suponía sólo el 1,7\% del total, a pesar de que en algunas comarcas del sur y del interior era del 20\%. Analógicamente la demografía presenta una tendencia acusada de envejecimiento poblacional debido al efecto conjunto de la baja natalidad y a la elevada masculinidad. En contraste, el número de municipios catalanes de menos de 2000 habitantes (frontera censal de la ruralidad) sólo ha cambiado ligeramente: de algo más de 700 poblaciones en 1970 a las 630 de $2004^{19}$.

15. GARCÍA RAMÓN, M.D; TULLA, A y VALDOVINOS, N., Geografía rural, Síntesis, Madrid: 1995, p. 31. ALDOMÀ, I. Amb el permís de Barcelona, Pagès, Lleida, 1999, p. 9.

16. KAISER, B., op. cit.

17. LITTLE, J. y AUSTIN, P., op.cit.

18. ESTALELLA, H. y CARRASCO, S. "La Catalunya Rural Contemporània” en S. GINER, (dir.) La societat catalana, Inst. d'Estadística de Catalunya, Barcelona, 1998, pp. 374 y ss.

19. TULLA, A.”El món rural i la diversitat de les persones. Canvis demogràfics i integració social al món rural”, 
En cambio, los niveles educativos (porcentaje de titulados medios y superiores) son iguales, y en ciertos casos superiores, a los de los ámbitos urbanos. Por otra parte, ni en términos de renta familiar, de estructura del mercado de trabajo o de especialización económica parece que la contraposición de una Cataluña rural con una Cataluña urbana tenga sentido. Es decir, mundo rural y mundo urbano son realidades macroeconómicas y demográficas diferenciadas, pero con una diversidad y complejidad internas que contribuyen a diluir sus respectivas fronteras ${ }^{20}$.

Por último, si pasamos de la perspectiva estructural al punto de vista de los actores sociales y, por ejemplo, centramos nuestro enfoque en la vida cotidiana de las personas, la separación entre ambos mundos todavía parece más borrosa. Tal y cómo afirma Sampedro:

El medio rural asiste a un verdadero trasiego de gente que viene y va, para trabajar, para descansar o divertirse, para estudiar, para veranear o invernar, durante la semana o el fin de semana, o en vacaciones... Pueblos que se llenan o vacían siguiendo el ritmo de la vida social. Trabajar fuera y volver, al final de la jornada. Vivir en la ciudad y echar la jornada en el campo. Estudiar en la ciudad y volver el fin de semana, o por las fiestas, o en verano. Trabajar en la ciudad y volver al cabo de los años porque tu chica o tu chico sea del pueblo - o del pueblo de al lado- y surge la posibilidad de construir un proyecto laboral y de pareja 'cono base' en el pueblo o en la cabecera de comarca. ${ }^{21}$

Esta movilidad, a la que propiamente denominamos "sedentarismo nómada" 22 , es característica de las sociedades hipermodernas, donde los agentes sociales se desplazan sin descanso por una multitud de lugares (hogar, trabajo, compras, vacaciones, segundas residencias, familia extensa...) como si fueran múltiples microespacios vitales. Desplazamientos físicos (a pie, en coche, en avión...), pero también movilidad electrónica o telecomunicativa: teléfonos fijos o móviles, correo electrónico, medios de comunicación... En esta nueva sociedad itinerante, de modernidad líquida, los procesos de rururbanización y, sobre todo, el incremento sin precedentes históricos de la movilidad social (geográfica, ocupacional, telecomunicativa...) de los actores sociales deja definitivamente obsoletas las concepciones esencialistas de aquello que es ser rural o urbano. Desde esta nueva perspectiva, lo rural ya no es un mundo aparte, a pesar de que tampoco es un mundo ilusorio que ya no existe, sino una categoría socialmente construida ${ }^{23}$.

Según esta nueva ruralidad, estaríamos en presencia un arraigo instrumental que trata de aprovechar el mejor de los dos mundos ${ }^{24}$, se podría para decir retomando el título del artículo de R. Sampedro: "Ser moderna y de pueblo, a la vez".

Primer Congrés del món Rural a Catalunya, Fundació Món Rural, Dep. d’Agricultura, Ramaderia i Pesca, Generalitat de Catalunya, Barcelona: 2006, pp. 317-357.

20. BONAL, R. “Del món rural al món urbà a Catalunya”. En GINER, S. (dir.) La societat catalana Institut d'Estadística de Catalunya, Barcelona, 1998, pp.351-366.

Col.lectiu CCB, opcit, 2006, pp. 450-451.

ALABART, A. y VILÀ, G. “Territori i estructura social” En MONTAGUT, M. (coord.). Societat catalana: Associació Catalana de Sociologia, Barcelona, 2007 p.199.

21. SAMPEDRO R., op. cit, 2008, p.181.

22. BERICAT E., Sociología de la movilidad espacial, C.I.S., Madrid, 1994.

23. DÍAZ MÉNDEZ, C., "Aproximaciones al arraigo y al desarraigo femenino en el medio rural” Papers no 75, 2005, pp. 63-84.

24. SAMPEDRO, R. op. cit., p. 188 
Este contexto de nueva ruralidad representa un reto más para la escuela y las administraciones deben dar una respuesta adecuada que satisfaga las necesidades y las aspiraciones de los alumnos y de sus familias que habitan en las zonas menos pobladas.

\section{La escuela rural en la cataluña de hoy}

En las zonas de población dispersa, en las que hay pocos alumnos, existe globalmente tres modelos de escolarización: a) concentración escolar en poblaciones más grandes, lo que conllevala necesidad de transporte escolar; b) pequeńas escuelas que acojan a los alumnos de los pequeños pueblos; y c) un modelo mixto de agrupación de los alumnos en un centro cercano a su residencia.

En Catalunya, se ha optado por mantener abiertas las escuelas de los pueblos que tengan un mínimo de 10 alumnos y si hay alumnos que viven en pequeñas aldeas que no tienen capacidad para tener una escuela, integrarlos en la escuela más cercana.

Cuando hablamos de escuela rural, hablamos únicamente de escuela primaria en donde las agrupaciones del alumnado se corresponden, por regla general, con la edad de los alumnos. Así pues, en la escuela urbana se dispone de una unidad para cada nivel educativo, de infantil y primaria, desde P-3 (3 años) hasta $6^{\circ}$ curso (once-doce años), según el número de alumnado, en muchos casos en un centro hay más de un grupo-clase por cada nivel.

En las escuelas rurales, debido al reducido número de niños y niñas, no llegan a tener una unidad para cada nivel educativo de forma que un mismo maestro atiende a alumnas y alumnos de distintos cursos en una misma aula.

La Conselleria d'Educació de la Generalitat define la escuela rural como aquella que es única en el pueblo, con una estructura variable, que se encuentra en un medio rural y que no llega a tener un grupo para cada nivel.

Tradicionalmente se había definido la escuela rural como la de las tres "p": pequeña, pública y de pueblo. Sea pequeña o no, sea de pueblo o no, la característica que hace singular a una escuela rural es que acoge y da servicio a las hijas e hijos de todas las familias de una comunidad, independientemente de los niveles culturales, económicos o sociales de cada una de las familias ${ }^{25}$.

Para favorecer la organización pedagógica, las escuelas se organizan estructuralmente en Zonas Escolares Rurales (ZER).

La ZER es una institución escolar formada por escuelas rurales. Se la considera como un centro único que respeta la identidad de las escuelas que la integran con la finalidad de avanzar colectivamente hacia una enseñanza de calidad y favorecer el éxito educativo del alumnado. Las escuelas integradas en una ZER son cercanas entre ellas y comparten similitudes socio-geográficas al mismo tiempo que comparten un mismo Proyecto educativo, las mismas normas de organización y funcionamiento y un mismo programa que ha de respetar la singularidad de cada una de las escuelas. ${ }^{26}$

25. TOUS J.-Ll. "Proposta de definició d'eseola rural", in L'escola díns el món rural, IV Jornades d'Escala Rural (Valls, abril de 1984), Bellaterra, ICE de la UAB, 1985, p. 146.

26. Documents per a l'organització i la gestió dels centres. Escoles rurals i zones escolars rurals, Departament d'Ensenyament, Generalitat de Catalunya, Barcelona, 2015. 
Las ZER nacieron con la intención de paliar las carencias y necesidades que tenían las escuelas y los docentes de los pueblos pequeńos, como por ejemplo: las excesivas materias, áreas, y cursos para un solo maestro y la falta de una ratio oficial maestro/alumno por escuela rural; la dificultad para encontrar metodologías y técnicas que den respuesta, de manera generalizada al hecho de tener más de un curso por clase simultáneamente; la escasa dotación de material en las pequeñas escuelas; la excesiva responsabilidad para un solo maestro, que tiene que dominar las materias que necesitan un considerable nivel de especialización, etc.

El número de escuelas de cada ZER es variable (de dos a cinco, la mayoría tiene tres) en función de su proximidad geográfica.

En el curso 2014-2015, había 278 escuelas integradas en 88 ZER, que atendían a 11.048 alumnos. En cuanto a su localización, veamos en el mapa siguiente la situación de las escuelas rurales.

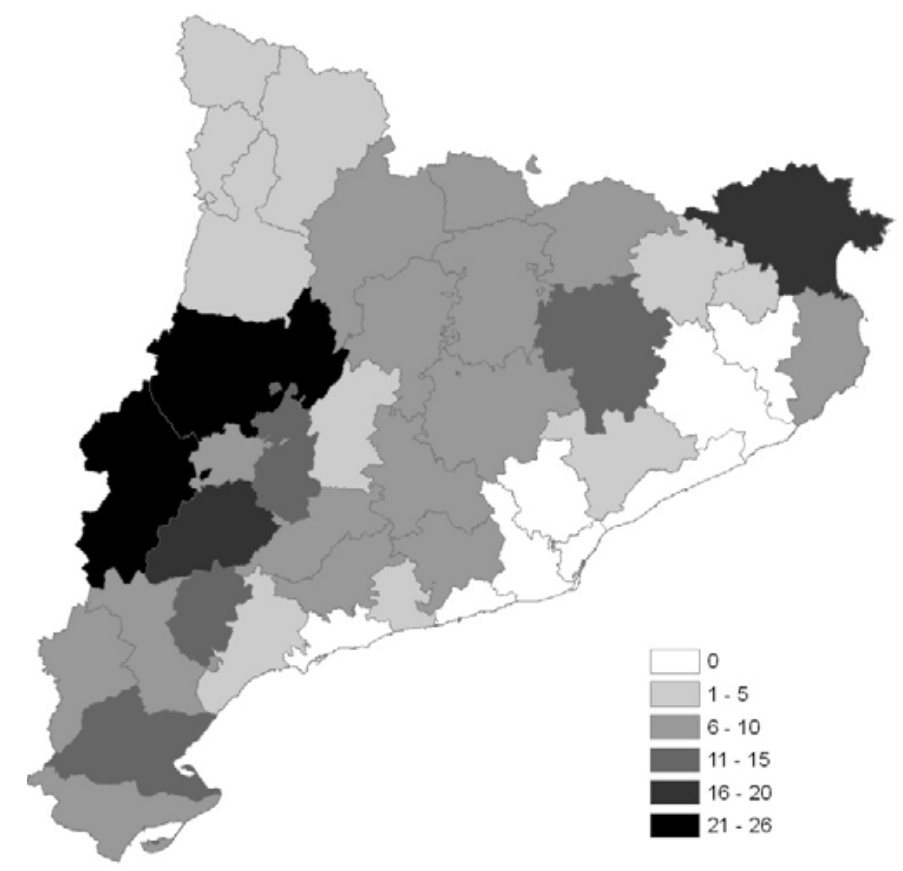

Mapa 2: Número de escuelas rurales en 2013. Fuente: Elaboración propia.

Si comparamos los mapas 1 y 2, observamos que las comarcas que no tienen ninguna escuela rural se corresponden con las zonas de mayor población urbana. Debemos, no obstante, relativizar el número de escuelas rurales en el noroeste, que es la zona con menor población y donde la mayoría de las escuelas son rurales, pero justamente como consecuencia de la poca población hay lógicamente menos escuelas. Lo contrario ocurre en el oeste y en el norte, zonas más pobladas, pero donde la población es más dispersa y hay un mayor número de escuelas rurales.

Por otra parte, el número es variable, el aumento o disminución en un periodo corto de tiempo del número de escuelas rurales puede explicarse por dos fenómenos antagónicos: el primero, el cierre de escuelas por falta de alumnos y el segundo porque ha aumentado el número de alumnos y escuelas que habían sido rurales pasan a ser 'graduadas completas' (con un aula para cada curso) y pierden la condición de escuela rural. 
En lo referente al número de alumnos en las escuelas rurales, una encuesta realizada en $2013^{27}$ muestra que dos de cada cinco centros tienen entre 25 y 50 alumnos, los centros que tienen entre 51 y 100 alumnos y los que tienen menos de 25 alumnos, representan tres de cada diez. En su gran mayoría los alumnos son autóctonos de la comarca, no obstante hay alumnos de padres inmigrantes y de neo-rurales.

En lo que concierne al número de aulas, la media es de 3,5 aulas por escuela y puede haber diferentes combinaciones cada año en función del número de alumnos, de su edad y de los docentes que haya en el centro, siendo un ejemplo más de la flexibilidad característica de la escuela rural.

El númerode docentes está, lógicamente, relacionado con el número de alumnos que hay en el centro. Hay una minoría de escuelas de menos de 25 alumnos (las más pequeñas) que tiene un solo docente, mientras que en los centros "más grandes" (entre 51 y 100 alumnos) disponen de más de cinco profesores. La organización de la escuela rural en centros integrados en una ZER prevé la existencia de profesores itinerantes especializados en la enseñanza de idiomas, educación física, educación musical, educación especial — para alumnos con algún tipo de carencia_-..., de esta forma, los alumnos de las escuelas rurales tienen los mismos docentes especialistas que los alumnos de los centros urbanos.

En España, todos los maestros y las maestras tienen la misma formación y las mismas condiciones económicas, con independencia del tipo de centro en el que ejerzan su docencia. En la escuela rural encontramos docentes de todas las edades, para algunos es un primer destino y cuando tengan la oportunidad pedirán el traslado a poblaciones más grandes, pero para otros — ocho de cada diezes una opción profesional a medio o a largo plazo, con lo cual la escuela rural cuenta con docentes experimentados en este tipo de escuelas, se garantiza la estabilidad de las plantillas y se facilita su integración en la población, aunque no exista la obligación de residir en la misma localidad en la que se ejerce la docencia. Otro hecho que cabe citar es que en Cataluña, la enseñanza es una profesión feminizada y la escuela rural no es una excepción. "En la enseńanza primaria el profesorado catalán está mucho más feminizado que en el resto de España, incluso más que en el conjunto de la OCDE [...] en la enseńanza primaria la tasa media de feminización en 2003 es del $80 \%$ en la OCDE, y del $71 \%$ en Espańa, pero del $85 \%$ en Catalunya, tanto en el sector público como en el privado" 28 .

En España, y por consiguiente en Cataluña, los programas de estudios en las enseñanzas infantil, primaria y secundaria obligatoria presentan algunas particularidades, ya que no existe un programa de estudios idéntico para todos los centros. El punto de partida es el curriculum que está estructurado en tres niveles:

a) La Administración educativa (Estado y Regiones autónomas) fija los objetivos, competencias, capacidades y contenidos mínimos, para todos los alumnos, es lo que en nuestro caso se conoce como el "primer nivel de concreción". Éste es abierto y flexible.

b) A partir de la idea de un curriculum abierto y flexible, cada centro debe adaptar el primer nivel de concreción a sus peculiaridades, a las características de sus alumnos, al entorno físico y social,... es lo que se llama "segundo nivel de concreción", que puede ser distinto en cada centro lo que permite que los centros tengan una cierta singularidad.

c) El docente será quien desarrolle —en el aula con sus alumnos - un "tercer nivel de concreción”, que consiste en llevar a la práctica el segundo nivel de concreción del curriculum para que los alumnos adquieran tanto los conocimientos, las competencias, las capacidades, las

27. Encuesta realitzada por L. Samper, T, Sala, y X. Burrial, a 202 escuelas rurales. Pendiente de publicación.

28. PEDRÓ, F, (dir), El professorat de Catalunya, Fundació Bofill, Barcelona, 2008, pp. 96 i 97. Nuestra traducción. 
habilidades, y las aptitudes que permitan alcanzar los objetivos del ciclo educativo ${ }^{29}$ llevando a cabo la adaptación pedagógica necesaria a las características de los alumnos.

Un curriculum abierto y flexible parte de un paradigma distinto al de un programa oficial cerrado y rígido, no obstante hay que tener presente que existe la obligatoriedad para los centros de cumplir con los objetivos mínimos fijados por la Administración educativa en el primer nivel de concreción, asegurando de este modo el progreso de todos los alumnos en su trayectoria escolar.

La apertura y flexibilidad que permite el curriculum es una ventaja para la escuela rural, puesto que en una misma aula encontramos simultáneamente alumnos de distintos niveles, es lo que se conoce como "aulas multinivel". En la escuela rural, la organización del tiempo - horario, calendario escolar,... i de la programación exigen una visión global de la composición concreta del grupo clase, a la vez que una concepción global y transversal tanto de los contenidos (programa) como de competencias y adquisición de habilidades, capacidades o aptitudes.

J.-L. Tous ${ }^{30}$, cita algunos aspectos diferenciadores de la escuela rural y por extensión de las "aulas multinivel": a) una heterogeneidad enriquecedora, con grupos formados por alumnos de diferentes edades, con capacidades y personalidades distintas; b) una dinámica interna positiva que propicia de manera natural y espontánea una dinámica vivencial entre el grupo clase y favorece el ejercicio, la adquisición de conocimientos, actitudes y hábitos como la solidaridad, la cooperación, el comportamiento respetuoso; c) permite la progresión personal individualizada, el alumno que presenta dificultades puede recuperar o reforzar sus carencias sin moverse del grupo de los compañeros con los que comenzó la escolarización, pero al mismo tiempo potencia la progresión de aquellos alumnos que destacan en capacidades y destrezas; el aula multinivel posibilita la adquisición de conocimientos y hábitos más allá de los que asigna el diseño curricular de base; d) potencia el ejercicio de la autonomía personal; el maestro tiene que atender a varios grupos; en un aula multinivel siempre hay alguien que trabaja de manera autónoma, bien sea en grupo o individualmente; e) es una práctica de la solidaridad cercana, precisamente porque el docente no puede atender a todos a la vez, de tal forma que el alumno que está al lado se convierte a menudo en apoyo y guía; $\mathrm{f}$ ) permite el uso de las metodologías, procedimientos y técnicas más diversas, en este sentido las TIC son idóneas para este tipo de escuela.

Los docentes de las escuelas rurales deben afrontar una serie de dificultades. Entre otras, un primer aspecto es la programación puesto que hay que secuenciar los contenidos y preparar las actividades para alumnos de distintos cursos, lo cual exige un buen conocimiento del curriculum. Paralelamente al conocimiento del programa oficial es necesario conocer la estructura interna del grupo clase del que se es responsable. No hay un grupo tipo, cada aula multinivel tiene una composición genuina, única y es imprescindible que el/la maestro/a la conozca muy bien. Un tercer punto a tener en cuenta es la elaboración de materiales, ya que los libros de texto están pensados principalmente para su uso con alumnos en un aula con un solo curso. No obstante, la estructura escolar de las Zonas Escolares Rurales facilita mucho la tarea de quienes llegan de nuevo a la escuela rural. La ZER puede ser un apoyo importante para los docentes que se incorporan de nuevo, ya que les puede facilitar programaciones y materiales que han sido pensados y realizados por quienes les han precedido.

29. Entendemos por "ciclo" un periodo más amplio que un año escolar. En la Educación primaria existe tres ciclos —inicial, medio y superior - con una duración de dos cursos cada uno.

30. TOUS, J-L., "L'escola arrelada al territori: l'escola comprensible, la pedagogía vivencial", Seminario con alumnos de la Facultat d'Educació de la Universitat de Lleida, octubre de 2014. No publicado.

Sobre las aulas multiniveles, véase también: Boix Tomás, R., Estrategias y recursos didácticos en la escuela rural, Barcelona, Graó, 1995. 
Como hemos visto, la docencia en una escuela rural presenta algunas particularidades y cuando preguntamos a los maestros y maestras de las escuelas rurales cuales son los puntos fuertes de este tipo de escuelas en Cataluña, señalan por orden de importancia los siguientes: la atención personalizada y la facilidad de las relaciones; la baja ratio profesor/alumno; la no masificación de la escuela; el agrupamiento por edades, que favorece el aprendizaje; el clima de clase favorable, con un ambiente familiar y de convivencia; la buena relación entre familias y maestros, el entorno medio-ambiental agradable y tranquilo, la relación entre la escuela y el pueblo, con las autoridades, pero también con sus habitantes, tengan o no hijos o hijas en la escuela. A nivel profesional valoran igualmente la autonomía docente. En términos generales, el curriculum es más flexible, los alumnos son más autónomos y es frecuente ver a los mayores que ayudan a los más pequeños, también existe una implicación de la comunidad con la escuela, "es 'mi' escuela".

Lo que por una parte se aprecia como puntos fuertes, por otra parte aspectos muy parecidos pueden ser percibidos como puntos débiles, como si fueran dos caras de una misma moneda. De esta forma, la soledad del niño en el curso o en el cicló, la existencia de diferentes niveles en la misma aula o un entorno muy reducido son los puntos débiles que enumeran principalmente. Algunos profesores se quejan de las dificultades que tienen para programar y del trabajo excesivo que conlleva la docencia en una escuela rural.

A nivel de infraestructuras, la escuela rural dispone de un equipamiento equiparable a la escuela urbana, no obstante en algunas escuelas — sobre todo en las que tienen más de 25 alumnos- se señala como deficiencias la falta de espacio, sobre todo la falta de aulas especializadas y de aulas polivalentes, pero también la falta de equipamiento para el deporte o lo pequeños que son los patios de recreo.

Los maestros valoran positivamente la existencia de las ZER y el hecho de realizar actividades conjuntas con centros similares, sin perder las escuelas su autonomía como centros. Los aspectos que más destacan son: el compartir maestros itinerantes, proyectos, experiencias, problemas,... o bien optimizar recursos materiales; asimismo el trabajo cooperativo entre los docentes de la ZER facilita que el docente no quede aislado.

\section{Reflexiones finales}

Actualmente, la escuela rural tiene las condiciones para ser un lugar privilegiado para la socialización y para el aprendizaje, dado el número reducido de alumnos. Esta situación permite la adquisición de conocimientos de una manera más individualizada y personalizada, así como la facilidad de establecer unas relaciones más fluidas entre los diversos actores de la comunidad educativa.

Podríamos decir que tradicionalmente la escuela rural ha sido "la Cenicienta" del sistema educativo $y$ han sido necesarios muchos esfuerzos por parte de los maestros que se han implicado porque han creído en este modelo de escuela y han defendido sus ventajas. El hecho de tener que superar los estereotipos de una escuela atrasada y con carencias, de tener que defender sus proyectos educativos y de reivindicar su existencia frente a unas políticas educativas que tendían a la globalización y a la concentración, en un momento en el que el modelo urbano era preponderante, ha representado muchas veces un gran reto.

Es satisfactorio comprobar que la escuela rural actual goza de una opinión positiva por su capacidad de ofrecer unas posibilidades formativas y educativas de calidad, sin embargo, conviene 
conocer cuáles son sus puntos fuertes y puntos débiles para no caer en un optimismo o un pesimismo excesivos.

La flexibilidad, el sentimiento de pertenencia, la implicación de la comunidad con la escuela, la implicación de los docentes, los medios materiales y humanos de los que dispone han resultado claves en la calidad educativa de este modelo minoritario que goza de buena salud pero que puede morir de éxito.

A nivel pedagógico y de organización, las ZER funcionan como una única escuela y este sentimiento de identidad se manifiesta también a niveles que van más allá de los aspectos organizativos, en aspectos que pueden parecer anecdóticos, pero que no dejan de tener un componente simbólico, por ejemplo al mostrar una imagen común, entre otras con una web para todas las escuelas o el papel de carta impreso y los chándales, que suelen ser los mismos para todas las escuelas de una ZER.

La escuela rural cumple con un cometido fundamental de escolarización de las zonas más despobladas del territorio o de las zonas donde la población es más dispersa con una calidad educativa equiparable a la de los centros situados en poblaciones más grandes o en las ciudades, evitando de este modo que los pueblos se queden sin los niños correteando por las calles.

Ejercer de maestro en una escuela rural no significa trabajar ni mejor ni peor que en cualquier otra escuela, sino trabajar de una manera diferente. Sin embargo, es necesaria una formación específica que generalmente no se da en la formación inicial de los maestros. 